\title{
Impact of egg production and stock structure on MSY reference points and its management implications for southern hake (Merluccius merluccius)
}

\author{
Santiago Cerviño ${ }^{\mathrm{a}, *}$, Rosario Domínguez-Petit ${ }^{\mathrm{b}}$, Ernesto Jardim $^{\mathrm{c}, \mathrm{e}}$, Sonia Mehault ${ }^{\mathrm{d}}$, Carmen Piñeiro ${ }^{\mathrm{a}}$, \\ Fran Saborido-Rey
}

\author{
a InstitutoEspañol de Oceanografía, Centro Oceanográfico de Vigo, Subida a Radio Faro, 36200 Vigo, Spain \\ ${ }^{b}$ Institute of Marine Research (CSIC), Eduardo Cabello 6, 36208 Vigo, Spain \\ ${ }^{c}$ Instituto de Investigaçao das Pescas e do Mar (IPIMAR), Lisbon, Portugal \\ d Institut Francais de Recherche pour l'Exploitation de la Mer (IFREMER), Lorient, France \\ e European Commission, Joint Research Centre, Institute for Protection and Security of the Citizen, Maritime \\ Affairs Unit G.04, TP 051, 21027 Ispra (VA), Italy \\ *: Corresponding author : Santiago Cerviño, tel.: +34 986492111 ; fax: +34 986498626 ; \\ email address : santiago.cervino@vi.ieo.es
}

\begin{abstract}
:
Spawning stock biomass (SSB) is one of the most common measures of stock status. However recent research on reproductive potential has shown that alternative variables may be an improvement over SSB. In the case of European hake (Merluccius merluccius), it is known that large individuals produce more eggs by unit of body weight, and their quality is better than those of small individuals. Under these circumstances, application of reproductive potential may be pertinent in the implementation of the Johannesburg agreement, since it is not only the spawning biomass but also its age or length structure that defines stock productivity and its ability to achieve maximum sustainable yield (MSY). In this contribution we used an agelength structured population model to assess the impact of different reproductive indices (total spawning biomass, female spawning biomass and egg production) on MSY reference points. First, we analyzed how these different indices alter our perception about per recruit productivity. Second, we analyzed the quality of these alternative reproductive indices to explain and predict recruitment using different model structures (Ricker and Beverton-Holt) and Bayesian inference. Third, we combined per recruit models and stochastic stock-recruitment relationships to estimate the probability distributions of MSY biological reference points (MSY, $F_{\text {msy }}$, Stock msy $_{\text {, and }} F_{\text {crash }}$ ). Our results show that, for hake stocks where larger fish have a strong contribution to stock reproductive potential, use of alternative reproductive indices will affect estimations of stock sustainability. The change in perception of relative contribution of each length class is exhibited in two opposite ways: reproductive potential per recruit and the shape of the stock-recruitment relationship defined with steepness. In our case, the change from SSB to egg production, resulted in an increase in steepness which counteracted the depletion per recruit resulting in a larger $F_{\text {msy. }}$.
\end{abstract}

Keywords: Egg production ; Biological reference points ; Steepness ; Length based models ; Hake

\section{Introduction}

The main outcomes of the Johannesburg Summit in 2002 for fisheries management was the decision to "maintain or restore depleted fish stocks to levels that can produce maximum sustainable yield (MSY) on an urgent basis and where possible by 2015". By "stock levels" management bodies and scientists frequently reference spawning stock biomass (SSB), which is one the most common measures of population health or reproductive potential used in fisheries. This measure has been criticised since other measures that consider fecundity (Lambert, 2008, Marshall et al., 1998 and Mehault et al., 2010), age dependent maternal effects (Berkeley et al., 2004 
Lucero, 2008) or diversity of population structure (Marteinsdottir and Thorarinsson, 1998) may be more appropriate. This criticism is caused by the arguable assumption that SSB is proportional to egg production which is not the case for many species where older fish produce relatively more eggs per unit body weight (Morgan et al., 2009; Mehault et al., 2010) and eggs of better quality than young fish (Birkeland and Dayton, 2005, Marshall et al., 2006). Trippel (1999) defines stock reproductive potential (SRP) as a measure that represents a stock's ability to produce viable eggs and larvae that may eventually recruit to the adult population or fishery.

Fisheries management makes use of indices and reference values to decide on the actions to take. The two most common references are fishing mortality $(\mathrm{F})$ that refers to the level of exploitation and SSB that refers to the biomass of adults. Biological reference points (BRP) are a set of reference values by which the status of the stock is assessed defining a particular state of the fishery and exploited population. BRPs can indicate a target aimed at by management (target reference points), or a limit such as a reference to a potential dangerous state that has to be avoided (limit reference points), e.g. SSB levels below which productivity is endangered. The Johannesburg Summit called for stocks to be restored to levels that can produce MSY by 2015 where possible. MSY is the maximum mean yield that an equilibrium population may produce. The level of fishing mortality that produces MSY is known as $\mathrm{F}_{\mathrm{msy}}$ and the corresponding level of biomass is known as $\mathrm{S}_{\mathrm{msy}}$ (Stock level able to produce MSY). EU policies are applied in order to reach $\mathrm{F}_{\mathrm{msy}}$ in 2015 and ICES provides advice to reach this target (ICES, 2011).

The above references, also called "MSY reference points", are linked through the exploitation pattern of the fishery and the stock productivity that is represented by a stock-recruitment 
relationship (Sissenwine and Shepherd, 1982). The problem is that stock-recruitment models (SR) are frequently difficult to fit to available data. To overcome this problem the scientific community has looked for MSY reference point proxies, among which those based on yield-perrecruit models (YPR) were initially the most popular, like $\mathrm{F}_{\max }$ (Beverton and Holt, 1957) or $\mathrm{F}_{0.1}$ (Gulland and Boerema, 1973). Alternative proxies are those based on the relative depletion of the stock as a function of fishing mortality, also on a per-recruit basis (Clark, 1991; Goodyear, 1993). These reference points opened the ground for integrating alternative measures of stock reproductive potential (Morgan et al., 2009; Spencer et al., 2007).

The difficulties in adjusting S-R models make the analysis of alternative measures of the stock reproductive potential (SRP) difficult to evaluate. The literature shows examples of "improved" reproductive potential measures that have little impact on improving the fit of model to observed data (Brunel, 2010, Marshal et al., 2006; Murua, 2010). This is an important issue to address when analyzing alternative measures. However, biological features like length at maturity or fecundity change our perception of the resilience of the stock by affecting S-R steepness (Myers et al., 2002) which has a large impact on reference points (Brooks et al., 2010).

Murawski et al. (2001) and Marshall et al. (2006) have found that when considering mechanisms that produce higher reproductive potential for larger fish, like increases in both relative fecundity and egg quality with body size, the use of SSB may produce overly optimistic fishing expectations in stocks with truncated age structures since SSB might overestimate the resilience of these stocks. Morgan et al. (2009) analysed eight different stocks with different biological characteristics and showed that biological reference points may be under or overestimated by 
alternative reproductive potential measures compared with SSB. Although they did not find consistency in which indices of reproductive potential are more conservative in terms of management. Spencer et al. (2007) analysing rockfish species did not find relevant differences when considering larval survival (old rockfish produce larvae with higher survival than young rockfish) compared with traditional SSB reference points. The authors explain this result based on two counteracting mechanisms when considering the positive function of parent age and larval quality. The first one is a stronger reduction of reproductive potential per recruit for the same F. This mechanism is counteracted with an increase in the perceived stock resilience. Both mechanisms operate in opposite directions thus partially cancelling their effects and thus their impact on alternative $\mathrm{F}$ reference points.

Hake (Merluccius merluccius) is a widely distributed species in the Northeast Atlantic, from Norway to Iceland $\left(62^{\circ} \mathrm{N}\right)$ and to Mauritania $\left(21^{\circ} \mathrm{N}\right)$. The Atlantic population supports one of the main fisheries in the Northeast Atlantic area. Currently the existence of two stocks is recognized, the northern and southern; the latter is distributed around the Atlantic coast of the Iberian Peninsula. Hake is a top predator and exhibits cannibalism (Velasco and Olaso, 1998; Mahe et al., 2007). Although there is important knowledge regarding hake growth based on tagging experiments (de Pontual et al., 2003; 2006; Piñeiro et al., 2007) and daily growth (Kacher and Amara, 2005; Piñeiro et al., 2008), there is no clear basis for age interpretation based on otolith rings. Sex ratio at length is skewed to males between $25-45 \mathrm{~cm}$ and afterwards the female fraction increases until about $70 \mathrm{~cm}$ when it reaches 100\% (Murua, 2010). Male hake mature at about $32 \mathrm{~cm}\left(\mathrm{~L}_{50}\right)$ meanwhile females mature at about $45 \mathrm{~cm}$ although there is high temporal variability for this parameter (Dominguez et al., 2008). Large hake produce more eggs per unit of 
weight and these are of better quality, i.e. larger size, and higher dry weight (Mehault et al., 2010) compared to small hake. The combination of these effects, delayed female maturity, skewed sex ratio and the increase of egg production with length, suggest hake is a species of special interest to be considered for evaluating stock reproductive potential and its implications for management.

Southern hake stock assessment is undertaken annually by the International Council for the Exploitation of the Sea (ICES) to provide scientific advice to the European Commission about exploitation levels. The assessment model used at the moment is Gadget (Begley and Howell 2004), an age-length structured forward-simulation model where fishing and biological processes are length dependent. The length based approach chosen for this stock was due to the ageing problems mentioned previously. The stock status reference is based on total SSB and $\mathrm{F}_{\max }$ is used as a target proxy for $\mathrm{F}_{\mathrm{msy}}(\mathrm{ICES}, 2010)$.

In this paper, we used an age-length structured population dynamic model to assess the impact of different reproductive indices (i.e. total spawning biomass, female spawning biomass and egg production) on MSY reference points. Firstly, we analyzed how these different indices alter our perception about per recruit productivity using YPR and stock reproductive-potential per-recruit (SPR) length based models. Second, we analyzed the ability of these alternative reproductive indices to explain and predict recruitment using Ricker and Beverton-Holt models as well as Bayesian inference. Third, we combined per recruit models and stochastic S/R to estimate the probability distributions of MSY biological reference points (MSY, $\mathrm{F}_{\mathrm{msy}}, \mathrm{S}_{\mathrm{msy}}$, and $\mathrm{F}_{\text {crash }}$ ). $\mathrm{F}_{\text {crash }}$ is a reference point representing the theoretical limit of exploitation that drives a stock to 
collapse, corresponding with the replacement line at the origin of the stock-recruitment relationship. Finally, a comparative statistical analysis among MSY reference points (including proxies) considering factors affecting them is performed to evaluate the sensitivity of MSY to alternative reproductive potential indices.

\section{Material and methods}

Different analyses were performed to estimate MSY reference points with four alternative reproductive potential indices: total SSB, female SSB and egg production under two different assumptions (constant or exponential extrapolation after $80 \mathrm{~cm}$ ). First we estimated the values for these four reproductive potential measures; second these trends were used to fit models that relate stock reproductive potential with recruitment (Beverton-Holt and Ricker S-R models); finally we use these model fits to estimate MSY reference points which were compared with MSY proxies like $\mathrm{F}_{\max }$ or proportion of virgin stock reproductive potential per recruit (\%SPR).

\subsection{Data}

Estimates of abundance $(\mathrm{N})$ and fishing mortality $(\mathrm{F})$ at length (1 to $129 \mathrm{~cm})$, by quarter, from 1982 to 2008 were provided by ICES (2010). The model was developed with GADGET (Begley and Howell 2004). F at length from landings and discards by quarter from the last 3 years were used to estimate a mean exploitation pattern. $\mathrm{N}$ at the beginning of the second quarter, near the main recruitment period (Murua, 2010), was used to calculate different reproductive potential measures. 
Biological data consisted of proportion of maturity at length (total and female from 1982 to 2008); sex ratio at length (from 1982 to 2008), weight at length, and fecundity at length (both were considered constant for the whole period). Weight at length was calculated from a length weight relationship (ICES, 2010). These data were collected by the Spanish and Portuguese sampling programmes, carried out by the Instituto Español de Oceanografía (IEO) and Instituto de Investigaçao das Pescas e do Mar (IPIMAR) respectively. After 2001 the data were collected under the framework of the European Data Collection Regulations (EC/1543/2000; $\mathrm{EC} / 199 / 2008)$.

Egg production at length was extracted from Mehault et al. (2010) for the first quarter and assumed to be constant along the time series. Batch fecundity (BF) was estimated by the gravimetric method and then $\mathrm{BF}$ at length $\left(\mathrm{BF}_{1}\right)$ was calculated. On the other hand, as European hake exhibits a protracted spawning season with the main peak during the first quarter, Spawning Fraction (SF) was estimated for this period and $\mathrm{SF}$ at length $\left(\mathrm{SF}_{1}\right)$ was calculated too as in Mehault et al. (2010). Realised fecundity was the product of the estimated $\mathrm{BF}_{1}$ by the estimated $\mathrm{SF}_{1}$ at length in the first quarter within the length range between 40 and $80 \mathrm{~cm}$. Fecundity data were scarce and their length range only covered from 40 to $80 \mathrm{~cm}$.

Biological information is summarized on Figure 1 and Table 1 showing the trends at length for the mean of last 3 years. Females mature at larger size than males, delaying the size of female maturity compared with total maturity (left panel). Female frequency increases at larger sizes, reaching $100 \%$ of the population at sizes beyond $70 \mathrm{~cm}$ (middle panel). Larger females produce 
relatively more eggs than smaller hake, so egg production by unit of weight is about 6 times higher at $80 \mathrm{~cm}$ than at $40 \mathrm{~cm}$ (right panel).

\subsection{Indices of reproductive potential}

Four indices of reproductive potential (RP) were calculated using the population's numbers-atlength estimated by the assessment model at the end of the first quarter and the biological information described previously. For comparison purposes the total spawning stock biomass $(S S B)$ was included in the analysis, together with the female spawning stock biomass ( $f S S B)$, the egg production with constant extrapolation $(E P c t)$ where egg production for fish larger than 80 $\mathrm{cm}$ was assumed to have the same fecundity as $80 \mathrm{~cm}$ fish, and the egg production with exponential extrapolation (EPexp) where fecundity for fish larger than $80 \mathrm{~cm}$ was assumed to increase following the model trend. Equations employed to compute RP indices are presented below.

$$
\begin{aligned}
& S S B=\sum_{l=1}^{130} N_{l} * W_{l} * \text { tMat }_{l} \\
& f S S B=\sum_{l=1}^{130} N_{l} * W_{l} * \text { fMat }_{l} * s \text { Rat }_{l} \\
& E P C t=\sum_{l=1}^{130} N_{l} * W_{l} * \text { fMat }_{l} * s \text { Rat }_{l} * f e c C t_{l} \\
& E P \exp =\sum_{l=1}^{130} N_{l} * W_{l} * f M a t_{l} * s \text { Rat }_{l} * f e c \operatorname{Exp}_{l}
\end{aligned}
$$


where sub-index $l$ stands for length, $N$ is the abundance, $W$ is the individual weight, $t$ Mat is the total maturity proportion, $f M a t$ is the female maturity proportion, $s R a t$ is the sex ratio, fecCt is the fecundity extrapolated constant and fecExp the fecundity exponentially extrapolated.

Note that all values (SSB, $f S S B, E P c t$ and EPexp) exhibit a sequential progression in the relative contribution of larger fish to the stock RP, with larger fish relatively most important in EPexp, followed by EPct, FSSB and finally SSB.

\subsection{Stock reproductive potential - recruitment models $(S-R)$.}

Two different S-R models have been implemented, Beverton-Holt (Eq. 5) and Ricker (Eq. 6), and a Bayesian approach was used to fit the models. The fits were performed with OpenBUGS (www.openbugs.info) and R scripts with the R library R2OpenBUGS. The five initial years of the recruitment series (1982-86) were not used for fitting the S-R due to doubts about their quality (ICES, 2010). Priors for S-R parameters were defined as "non-informative" having a minimum impact on the posteriors. Priors for Ricker (equation 5) are: $\log (\mathrm{a}) \sim$ normal $($ mean $=0$, precision $=0.001) ; \mathrm{b} \sim$ uniform $(\min =0, \max =2)$; model precision in log scale tau $\sim$ gamma (alpha $=1$, beta $=0.1)$. Priors for Beverton-Holt (equation 6) are: $\log (a) \sim$ normal $($ mean= 0 , precision $=0.001) ; \mathrm{b} \sim$ uniform $(\min =0, \max =1000)$; model precision in los scale tau $\sim$ gamma (alpha=1, beta=0.1). A thinning of 10 ( 1 every 10 draws was saved) and a burning of 1000 (the first 1000 draws were deleted) were set after analysis of prior $v s$. posterior plots; traces and autocorrelation. Trials with different seeds were set to check convergence. The four RP indices were fit with both S-R models giving a total of eight S-R fits. The final MCMC posterior distribution is made of 1000 values for each parameter in the eight models. 
$R=a * S * \exp (-b * S)$

$R=a * S /(b+S)$

Where $R$ is recruitment, $S$ the stock reproductive potential index (SSB, fSSB, EPct or EPexp) and $a$ and $b$ are the function parameters.

Steepness $(h)$ is defined as the fraction of recruitment from an unfished population obtained when the stock reproductive potential is $20 \%$ of its unfished level (Mangel et al., 2010). Note that we have changed referenced definition using "stock reproductive potential" instead of "spawning stock biomass". Steepness for all models was estimated from posterior S-R parameters. Stock reproductive potential per recruit at $\mathrm{F}=0\left(\mathrm{SPR}_{0}\right)$ was estimated according to equations 7 (Ricker) and 8 (Beverton-Holt).

$$
\begin{aligned}
& h_{r k}=0.2 * \exp \left(b * S_{0} * 0.8\right) \\
& S_{0}=\ln \left(S P R_{0} * a\right) / b \\
& h_{b h}=a * S P R_{0} /\left(4 * b+a * S P R_{0}\right)
\end{aligned}
$$

Where $h_{r k}$ and $h_{b h}$ are the steepness for Ricker and Beverton-Holt models respectively; $S_{0}$ is the population level at $\mathrm{F}=0$, i.e. equivalent to the virgin $\mathrm{SRP} ; S P R_{0}$ is the stock reproductive potential at $\mathrm{F}=0 ; a$ and $b$ are the $\mathrm{S}-\mathrm{R}$ parameters corresponding to equations 5 and 6.

\subsection{Length based Biological reference points}

YPR, independent of the stock reproductive potential, was firstly estimated. Afterwards, SPR and MSY reference points ( $M S Y, F_{m s y}, F_{c r a s h}$ and $\left.S_{m s y}\right)$ were estimated for each combination of 
$R P$ index and S-R model. Per recruit analysis was performed with a length based dynamics written in $\mathrm{R}$ programming language reproducing the GADGET dynamics defined for Southern hake in ICES (2010).

YPR was calculated for different levels of $F$ to estimate $F_{\max } . F_{\max }$ is the current proxy to $F_{\text {msy }}$ for this stock (ICES, 2010). SPR was calculated for the four different RP indices for a grid of F values from 0 to 2 . The biological reference points corresponding to a percentage of the virgin $\operatorname{SPR}(\mathrm{X} \% \mathrm{SPR})$ were also estimated.

To estimate the distribution of MSY reference points, the 1000 posterior parameters from each of the eight S-R curves were combined with per recruit estimations following Sissenwine and Shepherd (1987). Posterior equilibrium yield and stock curves under different levels of F were performed to calculate the posterior MSY reference point distribution.

\subsection{Relative contribution of RPs to the length stock structure}

The SPR algorithm was also used to estimate the relative contribution of different length classes to population productivity. The relative contribution depends on the biological features of different RPs and also depends on the F.

Abundance per recruit at length was estimated for three different levels of $F$ multipliers $\left(F_{\text {mult }}\right): 0$, 0.5 and $1 ; \mathrm{F}_{\mathrm{mult}}=1$ correspond to the status quo $\mathrm{F}(=0.7)$ (ICES, 2010). Equations 1 to 4 (without summing up along the lengths) were applied to estimate productivity at length for every RP at the three $\mathrm{F}$ levels. We produced 12 different curves ( $3 \mathrm{~F}$ levels times $4 \mathrm{RPs}$ ). To compare RPs 
with different units (biomass and number of eggs) all the curves were presented in relative scale, i.e. the abundance for every length class was divided by the total abundance in each curve. Mean length was estimated for every curve. Mean length was weighted with the RP contribution in each length class following the equation:

$$
L_{\text {mean }}=N_{l} * l / \sum_{l=1}^{130} N_{l}
$$

Where $N$ is the abundance and $l$ is the length group.

\section{Results}

The consideration of biological information to develop alternative RP indices has had different impacts on the Southern hake stock perception. These alternative RPs are: SSB, fSSB, EPct and EPexp, which follow a sequential progression in the contribution of larger individuals on population reproductive potential. The time series (Figure 2) do not show big changes in the trends of alternative indices and the linear correlation among them is always high (Figure 3 ). The inclusion of this biological information does not improve the quality of the S-R fit but the opposite (Figure 4 and Table 2). However there is a clear impact of this biological information on the shape of the function. Steepness increases along the RP sequence ( $S S B, f S S B, E P c t$ and EPexp) having a direct impact on our perception of stock resilience (Figure 5 and Table 2). The SPR curves show that for the same F, stock depletion (\%SPR) increases along the RP index sequence (Figure 6 and Table 3). When we combine the S/R curves with the SPR to estimate $\mathrm{F}_{\mathrm{msy}}$ reference points we found trends in the opposite direction than SPR analysis (Figure 7). 
Other MSY reference points (MSY and $\mathrm{F}_{\text {crash }}$ ) also showed clear trends along the sequence of indices (Table 3)

\subsection{Temporal trends}

Trends by year are presented in Figure 2. Recruitment at age 0 ranges between 50 and 100 million fish until 2004. Afterwards recruitment is over 100 million fish. Trends on the four RP indices are quite similar. Contrary to the recruitment, SSB is high at the beginning and low at the end of the time series. None of the other RP stock trends (fSSB, EPct or EPexp) show strong differences compared with SSB. Apparently, good recruitment observed in recent years can not be explained by the inclusion of more biological realism in the RP estimation.

Linear correlations among different trends are presented in Figure 3. Correlations are weak among all the RP indices and the recruitment ( 0) although high among different RP indices. As it was explained previously, the different indices considered a progressive increase of the contribution of larger fish from SSB to EPexp. As expected, indices with similar large fish contribution to reproductive potential were strongly correlated ( $\mathrm{r}$ between $0.96-1$ ); in contrast, lower correlation was observed between $S S B$ and $E P(0.92$ and 0.93$)$.

The analysis of RP trends and correlations did not show any important change of the new indices compared with the SSB.

\subsection{Stock-recruitment relationship}

$\mathrm{S}-\mathrm{R}$ fit is presented in Figure 4 for the four RP indices and the two stock recruitment curves. Data ranged from 1987 to 2008 and covered years of high fishing mortality, well over $F_{\max }$ 
(ICES, 2010). With these high mortalities only the three starting points (1987-89) were in an area of higher stock size. These three points define the overcompensatory trend in the Ricker curve making large differences on the expected recruitment at high stock levels compared with Beverton-Holt models. At low stock sizes the information was also scarce and did not provide any robust signal of recruitment reduction that could be used to estimate slope at origin. However slope at origin was always higher in Beverton-Holt models than in Ricker models.

Comparisons among stock-recruitment models estimated with various RP indices are presented in Table 2. For Ricker models, SSB and fSSB showed the best fit (compared with EPct or EPexp) with higher median $\tau$ (inverse of variance) and lower Deviance Information Criterion (DIC). For Beverton-Holt models results were not so clear and $\tau$ and DIC present similar figures. Among the two models fitted with four different RP indices, Ricker either fitted with SSB or fSSB were the best models in terms of DIC or $\tau$.

Figure 5 shows the distribution of S-R steepness $(h)$ for the eight scenarios; steepness increased with the sequence of RP indices. When large fish had more contribution (e.g. EPexp) the steepness was higher. Maximum steepness for Beverton-Holt is 1. Under both models the trend is the same, with larger steepness for EP than for SSB. For Ricker models median $h$ ranged from 4.42 (with SSB) to 8.74 (with EPexp). In Beverton-Holt models $h$ ranged from 0.96 (with SSB) to 0.99 (with EPexp) (Table 2)

\subsection{Biological reference points}

$F_{\max }$, which is the current $F_{\text {msy }}$ proxy (ICES, 2010) for management purposes was estimated at 0.24 and $F_{0.1}$ at 0.16 . YPR reference points $\left(F_{\max }\right.$ and $\left.F_{0.1}\right)$ are independent of different $R P$ 
indices. They are presented here as a reference when comparing with alternative MSY reference points.

Stock reproductive potential per recruit (SPR) reference points are presented in Figure 6 and Table 3. SPR was scaled to a maximum of 1 for all RP indices to facilitate the comparison among them. With the same F, SSB produces more SPR than fSSB, EPct and exponential EPexp. This means a higher depletion along the RP sequence. On the other hand, in order to reach the same relative stock depletion, 30\% SPR for instance, we should use different Fs, higher F for SSB, lower for FSSB; lower for EPct and even lower for EPexp. RP indices where large individuals have a low influence (SSB) are associated with a higher $\mathrm{F}_{\% \mathrm{SPR}}$ than those where larger fish have more weight, like EP.

Figure 7 shows boxplots for $1000 \mathrm{~F}_{\mathrm{msy}}$ for the four RP indices and the two S-R models as well as deterministic $35 \% \mathrm{SPR}$ and $\mathrm{F}_{\max }$ Beverton-Holt models produce $\mathrm{F}_{\mathrm{msy}}$ distributions with an upper limit in $\mathrm{F}_{\max }(0.24)$. The $\mathrm{F}_{\mathrm{msy}}$ Beverton-Holt boxplot distribution ranged from lower values for SSB $($ median $=0.23)$ to higher values for EPexp $($ median $=0.24)$. The same increasing trend was observed for Ricker, where $F_{\text {msy }}$ was always over $F_{\max }$, with a minimum median of 0.56 for SSB to 0.60 for EPexp. Both models presented the same trend for different RP indices with the lowest $\mathrm{F}_{\mathrm{s}}$ for SSB and sequential increases with fSSB, EPct and EPexp, respectively. This increasing trend is in the opposite direction than those observed for SPR (decreasing); see for instance $\mathrm{F}_{35 \% \mathrm{SPR}}$ (Figure 7) which resulted in F values of $0.20,0.18,0.16$ and 0.14 for SSB, fSSB, EPct and EP respectively. This raises the question about the percentage of SPR used to set F\%SPR as a proxi for $\mathrm{F}_{\mathrm{msy}}$ since the percentage that best represents $\mathrm{F}_{\mathrm{msy}}$ depends on the RP index used; see for example the differences between $\mathrm{F}_{35 \% \mathrm{SPR}}$ and Beverton-Holt $\mathrm{F}_{\mathrm{msy}}$ in Figure 7. 
The other reference points (MSY and $\mathrm{F}_{\text {crash }}$ ) also presented a clear trend although not always in the same direction (Table 3). The expected MSY presented big differences depending on the S-R model and RP index. Ricker MSY increased from SSB (14.8 kt) to EPexp (17.6 Kt). BevertonHolt MSY, which was always higher than Ricker MSY, decreased from $22.8 \mathrm{Kt}$ with SSB to

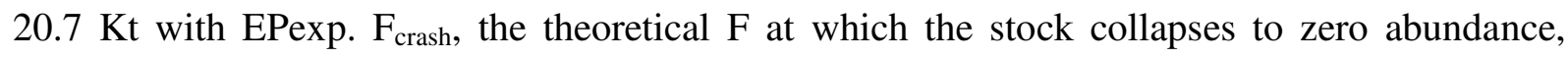
showed a decreasing trend along RP indices in both recruitment models. Ricker $\mathrm{F}_{\text {crash }}$ was always lower than Beverton-Holt $\mathrm{F}_{\text {crash }}$ and decreased from 1.21 (SSB) to 0.94 (EPexp) with the Ricker model and from 1.69 (SSB) to 1.49 (EPexp) with the Beverton-Holt model.

Notice that the distance between $\mathrm{F}_{\mathrm{msy}}$ and $\mathrm{F}_{\mathrm{crash}}$ was always shorter for Ricker than for BevertonHolt models. This distance, which is a measure of risk associated with $\mathrm{F}_{\mathrm{msy}}$ policies, showed a reduction trend along RP indices. The lower difference was observed for Ricker EPexp (median $F_{m s y}=0.6 ; F_{\text {crash }}=0.96$ ) and the maximum for Beverton-Holt $\mathrm{SSB}$ (median $F_{\mathrm{msy}}=0.23$ and $\left.\mathrm{F}_{\text {crash }}=1.69\right)$. Taking this measure as an indicator of risk, setting an $\mathrm{F}_{\mathrm{msy}}$ strategy presented a higher risk for Ricker than Beverton-Holt.

The $\mathrm{SPR}_{\mathrm{msy}}$ column in Table 3 presents the fraction of the stock relative to the pristine state when fishing at $\mathrm{F}_{\mathrm{msy}}$. In both cases (Ricker and Beverton-Holt) $\mathrm{SPR}_{\mathrm{msy}}$ became lower from SSB to EPexp indicating that the reduction from the pristine state at $\mathrm{F}_{m s y}$ is higher when considering RP where larger fish contribute most highly.

\subsection{Length relative contribution to population $R P$.}

Figure 8 shows the relative contribution of every length class to RP. This allows us to compare length-based RP contributions based on different units within each plot. However, we have to 
take into account that when $\mathrm{F}$ increases the absolute contribution of all length classes decreases for the same RP. Then, the curves from different panels are not comparable in absolute terms. Mean length is the parameter to compare curves from different panels.

In absence of fishing $\left(\mathrm{F}_{\mathrm{mult}}=0\right.$, in the left panel) the relative contribution of larger fish was more important than at higher F levels. EPexp presents the higher mean length, about $98 \mathrm{~cm}$, which decreases progressively for EPct, fSSB and SSB until $82 \mathrm{~cm}$. When F increased (Fmult $=0.5$ in the middle panel), the length structure was biased to lower size and mean length ranged between $81 \mathrm{~cm}$ (for EPexp) to $66 \mathrm{~cm}$ (for SSB). The same mean values should reduce more with the current $\mathrm{F}$ levels (Fmult $=1$; in the right panel), ranging from $72 \mathrm{~cm}$ (for EPexp) to $56 \mathrm{~cm}$ (for SSB).

\section{Discussion}

European hake is a dimorphic species for which males mature at a shorter length than females. This affects growth by sex and sex ratio at length (Murua, 2010) which is skewed towards females at larger fish size. This information (female maturity at length and sex ratio) enables an examination of female spawning biomass (fSSB) as an alternative to total spawning biomass (SSB). Because of the differences of maturity at length and sex ratio, fSSB brings to light the importance of larger individuals to stock reproductive potential (SRP). A step forward is the inclusion of fecundity, which leads to two additional alternatives SRPs (EPct and EPexp). Since hake egg production per unit of weight increases with length (Mehault et al., 2010; Murua, 2010), these two new indices (EPct and EPexp) complete the progressive sequence of four alternative indices in which larger fish are more important in stock productivity. This allows to 
evaluate the importance of considering additional hake biological information on biological reference points and also, in a more general way, to evaluate the role of demographic structure on biological reference points.

After having tried different alternatives to SSB RP indices (fSSB, EPct and EPexp) we have found that there are no relevant differences on temporal trends. Furthermore we did not find any improvement in the fit of the S-R relationship. Lack of improvement in S-R with more complex indices of RP also has been reported for American plaice (Hippoglossoides platessoides) and Greenland halibut (Reinhardtius hippoglossoides) (Morgan et al., 2011). This improvement depended on the amount of temporal variation in reproductive characteristics and on how well they are estimated (Morgan et al., 2011). Thus, if reproductive parameters do not change over time, then simple indices as SSB may be adequate. However, if temporal trends on these parameters are observed then it is more likely that more complex indices of RP will be better predictors of recruitment, as observed for Flemish Cap cod, where a longer and better quality time series was available (Morgan et al., 2011). Yet, our results showed that including more biological realism on the S-R relationship produced changes in steepness and consequently on the management reference points. This raises the question about how to balance biological realism and statistical consistency in order to choose the most appropriate index to use for management decisions. Marshall et al. (2006) suggest that the slight reduction in explanatory power that would accompany replacement of SSB must be judged against the benefit of correcting it for a systematic source of bias which may increase the risk of overexploitation. However in our work the opposite results were obtained, e.g. EP $F_{m s y}$ was higher than SSB $F_{m s y}$, meanwhile Marshall et al. (2006) found EP $F_{m s y}$ was lower than SSB $F_{m s y}$. Under this circumstance, moving from the current hake reference point, i.e. SSB, to a more biologically 
sound stock RP index, like EP, may be a risk since F increases. This risk seems difficult to bear if this more "reliable" RP index does not significantly improve the S-R model.

The S-R relationship is a key factor in the implementation of MSY policies. The quality of these models should be evaluated before justifying a change in the management RP reference. In this work the variability in the S-R relationship has been considered with no informative priors, and assuming no error in the RP indices, which may increase the uncertainty in the fit quality. A full evaluation of model fit should also include the error in the additional biological information (maturity, sex ratio and egg production) used to build alternative RP indices since this new information also adds uncertainty. Another problem fitting S-R models for European hake are the limited data available to estimate RP indices, being scarce at high and low stock sizes, and the high variability of observed recruitment in this range. This increases the difficulty in estimating S-R parameters, and especially the slope at origin and the level of compensation or overcompensation at high stock sizes. Informative S-R priors based on biological knowledge (Brooks et al., 2010, Myers et al., 2002) or based on the S-R performance from similar stocks (Myers et al., 2002) are alternatives that should be explored. Improvement of the S-R models could allow using them to estimate management reference points difficult to assume with the weak S-R model fit. For instance, Ricker $F_{m s y}$ is more than two times above Beverton-Holt $F_{m s y}$ and Ricker $F_{\text {crash }}$ is well below Beverton-Holt $F_{\text {crash }}$ (Table 3). If the Ricker model is more reliable (although uncertain), the risk of fishing at $\mathrm{F}_{\mathrm{msy}}$ increases in an unacceptable way and must be considered before defining a MSY strategy.

The results show that moving from SSB towards alternative RP indices where larger fish have relatively greater contribution increases $F_{m s y}$. This result is contrary to those of Murawski et al. 
(2001) stating that "traditional approaches to F-based reference points using SSB systematically overestimate the resiliency of stocks to fishing" and those from Marshall et al. (2006) who say “... using SSB as a measure of stock potential could lead to overly optimistic assessments of stock status". Morgan et al. (2009), performing similar analysis, found some stocks where $F_{m s y}$ increased with refined RP indices, although for some stocks $F_{m s y}$ decreased, with these differences being very high in some cases (e.g. Gulf of St Lawrence cod; SSB F $F_{\text {msy }}=0.14$ and EP $F_{m s y}=0.56$ ). Spencer et al. (2007) found that $F_{m s y}$ is relatively insensitive in rockfishes when considering reproductive indices where larvae from larger fish have a higher survival rate. These authors provide a thorough explanation for this weak sensitivity based on two counteracting mechanisms: (1) reduced SPR at the same F and (2) increase of perceived stock resilience. These two mechanisms explain why age-dependent maternal effects on rockfish spawning success may either increase or decrease $\mathrm{F}_{\mathrm{msy}}$. Although we have found different results than Spencer et al., (2007), with an increase in hake $F_{m s y}$, our results support their explanation. This rationale also explains why other authors (Marshal et al., 2006; Murawski et al., 2001) have reported different results as these depend on how the two processes are balanced in their stocks with alternative reproductive potential indices. Further research is needed to explain how to balance these two counteracting processes.

In the case of Southern hake analysed here, increasing refinement in RP indices produces a reduction of the $\%$ SPR for a given $\mathrm{F}$ (See for example 35\% SPR in figure 7). In contrast, we observed an increase of $F_{m s y}$ with the enhancement in RP indices. These opposite trends may be explained looking at $\%$ SPR fishing at $\mathrm{F}_{\text {msy }}$ (see SPRmsy in Table 3). The $\%$ SPR fishing at $\mathrm{F}_{\text {msy }}$ becomes lower as we move from SSB to EPexp. Such a result would support the pattern we have 
already identified for steepness and $\mathrm{F}_{\mathrm{msy}}$ because a lower value for \%SPRmsy indicates that the stock can be reduced further from unexploited conditions, i.e. the stock is more resilient

Alternatively we may explain the different trends in \%SPR and MSY reference points looking at the replacement lines. Replacement lines are straight lines that define the equilibrium point in the $\mathrm{S}-\mathrm{R}$ function for a given $\mathrm{F}$. For instance, $\mathrm{F}_{\max }$ (independent of SRP index) produces a different relative reduction of SPR values for every RP index. The inverse of this SPR is the slope of the replacement line in the S-R function and the point where the S-R function crosses the replacement line is the stock equilibrium point for $F_{\max }$. In the Beverton-Holt model $F_{\max }$ is the upper limit of $\mathrm{F}_{\mathrm{msy}}$ (Figure 7). This means that the slope of the replacement line corresponding with $\mathrm{F}_{\mathrm{msy}}$ is lower or equal than those corresponding to $\mathrm{F}_{\mathrm{max}}$. If steepness is one, then $\mathrm{F}_{\mathrm{msy}}$ equals $F_{\max }$ because equilibrium recruitment does not increase when reducing $F$ below $F_{\max }$. The difference between $F_{\max }$ and $F_{m s y}$ depends on the relative increase of equilibrium recruitment from $F_{\max }$, which depends on steepness. If $F_{m s y}$ is higher for a reproductive measure where $\% \mathrm{SPR}$ is lower for the same $\mathrm{F}_{\max }$ (see Figure 7) this means that the relative increase in equilibrium recruitment must be higher for this SRP and this depends on steepness and cannot happen if steepness is lower. Therefore, an increase in steepness is a condition needed to counteract the decrease in \%SPR. Since the change in SRP drives \%SPR and steepness in opposite directions and these two processes counteract each other when estimating $F_{\text {msy }}$, the differences in $\mathrm{F}_{\mathrm{msy}}$ with different SRP are relatively low.

An increase of hake $\mathrm{F}_{\mathrm{msy}}$ along the sequence of RP indices has been detected for Southern hake. If these results were supported by a clear improvement in the S-R fit, it would mean that including more biological realism in the hake assessment would allow us to increase the F target. 
That is because when larger fish are more productive, we need less of them to produce the same amount of recruits. Thus, the population can still be sustainable and maximally harvested with a greater F when larger fish have a lower relative presence. Similarly, faster rebuilding should be achieved if a greater proportion of larger fish are allowed to survive. However, without a clear improvement in the $S-R$ fit, moving towards a higher although more biologically sound $F_{m s y}$ (e.g. considering egg production), could increase the risk of overfishing. Under these conditions it is difficult to decide which RP index is more valid to be used as a management reference point. Fortunately there are alternatives to improve management of this stock with this new biological knowledge. Apart from changing the $\mathrm{F}$ target, if management measures protecting large females were developed we should expect an improvement of the performance of the fishery. These management measures could consider changes in exploitation pattern with mesh sizes, seasonal and/or spatial closures. Not taking into account that large hake are relatively more productive than small ones will bias the results and the potential improvement of these alternative management measures could not be clearly evaluated.

It is of major importance to include alternative RP indices in stock assessment and advice so that reproductive biology is explicitly taken into account, not only towards improving estimates but also to explore sources of uncertainty that are hidden and incorporating these uncertainties into scientific advice for management decisions.

\section{Acknowledgement}

We would like to thank FRESH (COST Action FA0601) which has provided a scientific platform to discuss and progress with this contribution. Comments from Liz Brooks, Paul Spencer and John Pope, the editor Edward Trippel, and two anonymous reviewers helped 
towards improving the manuscript. Funding for this research was received from the European Union's Seventh Framework Programme (FP7/2007-2013) under grant agreement $\mathrm{n}^{\circ}$ 244706/ECOKNOWS project. However, the paper does not necessarily reflect EC views and in no way anticipates the Commission's future policy in this area.

\section{References}

Begley, J., Howell, D. 2004. An overview of GADGET, the Globally applicable AreaDisaggregated General Ecosystem Toolbox. ICES CM 2004/FF, 13: 16.

Berkeley, S.A., Chapman, C., Sogard, S.M. 2004. Maternal age as a determinant of larval growth and survival in a marine fish, Sebastes melanops. Ecology 85: 1258-1264

Beverton, R.J.H., Holt, J.H.. 1957. On the dynamics of exploited fish populations. U.K. Minist. Agric. Fish. Fish. Invest. (Ser. 2) 19.

Birkeland, C., Dayton, P. K. 2005. The importance in fishery management of leaving the big ones. Trends in Ecology and Evolution, 20: 356-358.

Brooks, E. N., Powers, J. E., Cortés, E. 2010. Analytical reference points for age-structured models: application to data-poor fisheries. ICES J. Mar. Sci., 67: 165-175.

Brunel, T. 2010. Age-structure-dependent recruitment: a meta-analysis applied to Northeast Atlantic fish stocks. ICES Journal of Marine Science, 67: 1921-1939

Clark, W.G. 1991. Groundfish exploitation rates based upon life-history parameters. Can. J. Fish. Aquat. Sci. 48:734-750.

de Pontual, H., Bertignac, M., Battaglia, A., Bavouzet, G., Moguedet, P., Groison, A. L. 2003. A pilot tagging experiment on European hake (Merluccius merluccius): methodology and preliminary results. ICES J. Mar. Sci., 60: 1318-1327. 
de Pontual, H., Groison, A.L., Piñeiro, C., Bertignac, M. (2006). Evidence of underestimation of European hake growth in the Bay of Biscay and the relationship with bias in the agreed ageing method. ICES J. Mar. Sci. 63(9), 1674-1681.

Domínguez, R., Korta, M., Saborido-Rey, F., Murua, H., Sainza, M., Piñeiro, C., 2008. Analysis of the influence of stock structure and environmental changes in size at maturity for both European hake Atlantic populations. J. Mar. Sys. 71, 260-278.

Goodyear, C.P. (1993). Spawning stock biomass per recruit in fisheries management: foundation and current use. pp. 67-82. In: Smith, S.J., J.J. Hunt, and D. Rivard [ed.]. Risk evaluation and biological reference points for fisheries management. Can. Spec. Publ. Fish. Aquat. Sci, 120.

Gulland, J. A., Boerema, L.K.. 1973. Scientific advice on catch levels. Fish. Bull. 71: 25-335.

ICES. 2010. Report of the Working Group on the Assessment of Southern Shelf Stocks of Hake, Monk and Megrim (WGHMM), 5 - 11 May 2010, Bilbao, Spain. ICES CM 2010/ACOM:11. 571 pp.

ICES, 2011. Report of the ICES Advisory Committee, 2011. ICES advice 2011, Book 1.

Kacher, M., Amara, R. (2005). Distribution and growth of 0-group European hake in the Bay of Biscay and Celtic Sea: A spatial and inter-annual analyses. Fish. Res. 71, 373-378.

Lambert, Y. 2008. Why Should We Closely Monitor Fecundity in Marine Fish Populations? J. Northw. Alt. Fish. Sci., 41: 93-106.

Lucero, Y. 2008. Maternal effects and time to recovery. Bull. Mar. Sci, 83(1): 217-234.

Mahe, K., Amara, R., Bryckaert, T., Kacher, M., Brylinski, J.M. 2007. Ontogenetic and spatial variation in the diet of hake (Merluccius merluccius) in the Bay of Biscay and the Celtic Sea. ICES Journal of Marine Science 64: 1-10 
Mangel1, M., Brodziak, J., and DiNardo, G. 2010. Reproductive ecology and scientific inference of steepness:a fundamental metric of population dynamics and strategicfisheries management. Fish and Fisheries 11: 89-104.

Marshall, C. T., Needle, C. L., Thorsen, A., Kjesbu, O. S., and Yaragina, N. A. 2006. Systematic bias in estimates of reproductive potential of an Atlantic cod (Gadus morhua) stock: implications for stock- recruit theory and management. Can. J. Fish. Aquat. Sci., 63: 980-994.

Marshall, C. T., Kjesbu, O. S., Yaragina, N. A., Solemdal, P., Ulltang, O. 1998. Is spawner biomass a sensitive measure of the reproductive and recruitment potential of Northeast Arctic cod? Can. J. Fish. Aquat. Sci., 55: 1766-1783.

Marteinsdottir, G., Thorarinsson, K. 1998. Improving the stock-recruitment relationship in Icelandic cod (Gadus morhua) by including age diversity of spawners. Can. J. Fish. Aquat. Sci., 55: 1372-1377.

Mehault, S., Dominguez-Petit, R., Cerviño, S. Saborido-Rey, F. 2010. Variability in total egg production and implications for management of the southern stock of European hake. Fish Res. 104. 111-122.

Morgan, M.J., Murua, H., Kraus, G., Lambert, Y., Marteinsdóttir, G., Marshall, C.T., O’Brien, L., Tomkiewicz, J., 2009. The evaluation of reference points and stock productivity in the context of alternative indices of stock reproductive potential. Can. J. Fish. Aquat. Sci. 66, 404-414.

Morgan, M.J., Pérez-Rodríguez, A. and Saborido-Rey, F. 2011. Does increased information about reproductive potential result in better prediction of recruitment? Can. J. Fish. Aquat. Sci., 68: 1361-1368. 
Murawski, S. A., Rago, P. J., Trippel, E. A. 2001. Impacts of demographic variation in spawning characteristics on reference points for fishery management. ICES Journal of Marine Science, 58: 1002-1014.

Murua, H. 2010, The Biology and Fisheries of European Hake, Merluccius merluccius, in the North-East Atlantic. Advances in Marine Biology. 58: 97-154.

Myers, R.A., Barrowman, N.J., Hilborn, R. Kehler, D. 2002. Inferring Bayesian priors with limited direct data: applications to risk analysis. N. Am. J. Fish. Manage. 22, 351-364.

Myers, R.A., Mackenzie, B.R.. Bowen K.G., Barrowman N.J. 2001. What is the carrying capacity for fish in the ocean? A meta-analysis of population dynamics of north Atlantic cod. Can. J. Fish. Aquat. Sci. 58:1464-1476.

Piñeiro, C., Rey, J., De Pontual, H., Goñi, R. 2007. Tag and recapture of European hake (Merluccius merluccius L.) off the Northwest Iberian Peninsula: First results support fast growth hypothesis. Fish. Res. 88, 150-154.

Piñeiro, C., Rey, J., de Pontual, H., Garcia, A. 2008. Growth of Northwest Iberian juvenile hake estimated by combining sagittal and transversal otolith microstructure analyses. Fish. Res. 93, 173-178.

Sissenwine, M. P., Shepherd, J. G. 1987. An alternative perspective on recruitment overfishing and biological reference points. Can. J. Fish. Aquat. Sci., 44: 913-918.

Spencer, P.D., Hanselman, D., Dorn M. 2007 The effect of maternal age of spawning on estimation of Fmsy for Alaskan Pacific ocean perch. In: J. Heifetz, J. DiCosimo, A.J. Gharrett, M.S. Love, V.M. O’Connell \& R.D. Stanley (Eds) Biology, Assessment, and Management of North Pacific Rockfishes. pp. 513-33. Alaska Sea Grant, University of Alaska, Fairbanks. 
Trippel, E.A., 1999. Estimation of stock reproductive potential: history and challenges for Canadian Atlantic gadoid stock assessments. J. Northw. A. Fish. Sci.25, 61-81.

Velasco, F. and Olaso, I. 1998. European hake Merluccius merluccius (L., 1758) feeding in the Cantabrian Sea: seasonal, bathymetric and length variations. Fisheries Research 38: 33 44. 
Table 1. Summary of biological information for body length (grouped every $10 \mathrm{~cm}$ ), mean weight $(\mathrm{kg})$, total maturity (proportion), female sex ratio (prop.), female maturity (prop.), egg production exponential (eggs per gram) and egg production constant (eggs per gram)

\begin{tabular}{lcccccc}
\hline $\begin{array}{l}\text { length } \\
\text { group }\end{array}$ & $\begin{array}{c}\text { mean } \\
\text { weight }\end{array}$ & $\begin{array}{c}\text { total } \\
\text { maturity }\end{array}$ & $\begin{array}{c}\text { female } \\
\text { sex ratio }\end{array}$ & $\begin{array}{c}\text { female } \\
\text { maturity }\end{array}$ & EPexp & EPct \\
\hline $1-10$ & 0.00 & 0.00 & 0.40 & 0.00 & 0.00 & 0.00 \\
$11-20$ & 0.03 & 0.02 & 0.42 & 0.00 & 0.00 & 0.00 \\
$21-30$ & 0.13 & 0.11 & 0.50 & 0.00 & 0.10 & 0.72 \\
$31-40$ & 0.33 & 0.46 & 0.43 & 0.09 & 4.20 & 7.22 \\
$41-50$ & 0.69 & 0.87 & 0.58 & 0.61 & 11.95 & 11.95 \\
$51-60$ & 1.25 & 0.98 & 0.93 & 0.94 & 22.50 & 22.50 \\
$61-70$ & 2.05 & 1.00 & 1.00 & 0.99 & 35.83 & 35.83 \\
$71-80$ & 3.13 & 1.00 & 1.00 & 1.00 & 51.92 & 51.92 \\
$81-90$ & 4.54 & 1.00 & 1.00 & 1.00 & 70.77 & 59.95 \\
$91-100$ & 6.32 & 1.00 & 1.00 & 1.00 & 92.35 & 59.95 \\
$101-110$ & 8.52 & 1.00 & 1.00 & 1.00 & 116.65 & 59.95 \\
$111-120$ & 11.18 & 1.00 & 1.00 & 1.00 & 143.68 & 59.95 \\
$121-130$ & 14.17 & 1.00 & 1.00 & 1.00 & 171.83 & 59.95 \\
\hline
\end{tabular}

Table 2. Median values for posterior parameters $(a, b, \tau$ and $h) . a$ and $b$ are the stock-recruitment parameters; $\tau$ is the inverse of variance of the modelled recruitment (in $\log$ scale); $h$ is the steepness estimated with the Bayesian model; and Deviance Information Criterion (DIC) to compare model fit.

\begin{tabular}{lllllllllll} 
& \multicolumn{1}{l}{ Ricker } & \multicolumn{9}{c}{ Beverton-Holt } \\
\hline & $\boldsymbol{a}$ & $\boldsymbol{b}$ & $\boldsymbol{\tau}$ & $\boldsymbol{H}$ & DIC & $\boldsymbol{a}$ & $\boldsymbol{b}$ & $\boldsymbol{\tau}$ & $\boldsymbol{h}$ & DIC \\
\hline SSB & 15.12 & 0.0656 & 9.58 & 4.42 & 16.25 & 97.35 & 2.98 & 8.05 & 0.962 & 22.92 \\
fSSB & 19.50 & 0.0813 & 9.21 & 5.16 & 17.18 & 90.84 & 1.44 & 8.01 & 0.979 & 22.16 \\
EPct & 0.48 & 0.0018 & 7.44 & 6.78 & 22.19 & 85.96 & 34.56 & 7.95 & 0.990 & 21.52 \\
EPexp & 0.54 & 0.0021 & 7.75 & 8.74 & 21.25 & 86.47 & 33.52 & 7.99 & 0.993 & 21.71 \\
\hline
\end{tabular}

Table 3. Summary of reference points of four reproductive indices. SPR is F Stock Reproductive Potential Per Recruit at 3 levels of \% of depletion (30, 35 and 40\%). Ricker reference points (MSY in thousand tonnes, $F_{m s y}$ and $F_{\text {crash }}$ ) and the same Beverton-Holt reference points. $S_{\text {PR }}$ ms refers to proportion of SPR corresponding to $\mathrm{F}_{\mathrm{msy}}$. Ricker and Beverton-Holt figures are posteriors medians.

\begin{tabular}{|c|c|c|c|c|c|c|c|c|c|c|c|}
\hline & \multicolumn{3}{|l|}{ SPR } & \multicolumn{4}{|c|}{ Ricker } & \multicolumn{4}{|c|}{ Beverton-Holt } \\
\hline & $30 \%$ & $35 \%$ & $40 \%$ & MSY & Fmsy & Fcrash & SPRmsy & MSY & Fmsy & Fcrash & SPRmsy \\
\hline SSB & 0.23 & 0.20 & 0.17 & 14.84 & 0.56 & 1.21 & 0.10 & 22.81 & 0.23 & 1.69 & 0.31 \\
\hline FSSB & 0.21 & 0.18 & 0.16 & 15.68 & 0.58 & 1.05 & 0.07 & 21.59 & 0.23 & 1.55 & 0.28 \\
\hline EPexp & 0.16 & 0.14 & 0.12 & 17.64 & 0.60 & 0.94 & 0.03 & 20.72 & 0.24 & 1.49 & 0.19 \\
\hline
\end{tabular}




\section{List of figures}

Figure 1. Biological information used to estimate biological reference points (BRPs). Maturity proportion in the left panel, total (solid line) and female (dashed line). Sex ratio in the middle panel; mean (solid line) and historic values (black dots). Egg production (eggs / gr) in the right; observations only covers 40$80 \mathrm{~cm}$ range, outside this range two options were performed: extrapolation of the exponential fit (solid line) and constant (dashed line).

Figure 2. Trends by year from 1987 to 2008. Recruitment in upper plot. SSB (solid line) and Female SSB (dashed line) in the middle plot. Egg production under two different assumptions (lower plot); exponential (dashed) and constant (solid) after $80 \mathrm{~cm}$.

Figure 3. Pairs plots among the four reproductive potential indices (SSB, FSSB, EPexp and EPct) and recruitment (rec). Cells over the diagonal show the correlation coefficient between pairs.

Figure 4. Bayesian stock recruitment fit of four reproductive potential measures and two stock recruitment curves. Beverton-Holt model (dashed line) and Ricker model (solid line).

Figure 5. Box plot for steepness in Ricker (upper plot) and Beverton-Holt models (lower plot).

Figure 6. Stock per recruit for SSB, fSSB, EPct and EPexp scaled to a SPR=1 at F=0. Vertical line represents $F_{\max }$, equal to 0.24 and horizontal lines represent three levels of SPR (30, 35 and $40 \%$ )

Figure 7. Box plot for $\mathrm{F}_{\mathrm{msy}}$ with different reproductive potential measures (SSB, fSSB, EPct and EPexp). With Ricker model and Beverton-Holt model. $\mathrm{F}_{\max }$ and 35\%SPR are also presented.

Figure 8. Relative contribution of different length classes to RP population productivity at three different $\mathrm{F}$ levels. $\mathrm{F}_{\text {mult }}=1$ is equivalent to current $\mathrm{F}$ (ICES, 2010). $\mathrm{F}_{\text {mult }}=0.5$ is half the current $\mathrm{F}$. Horizontal lines represent the mean length of the population weighted with the RP. 
Figure 1
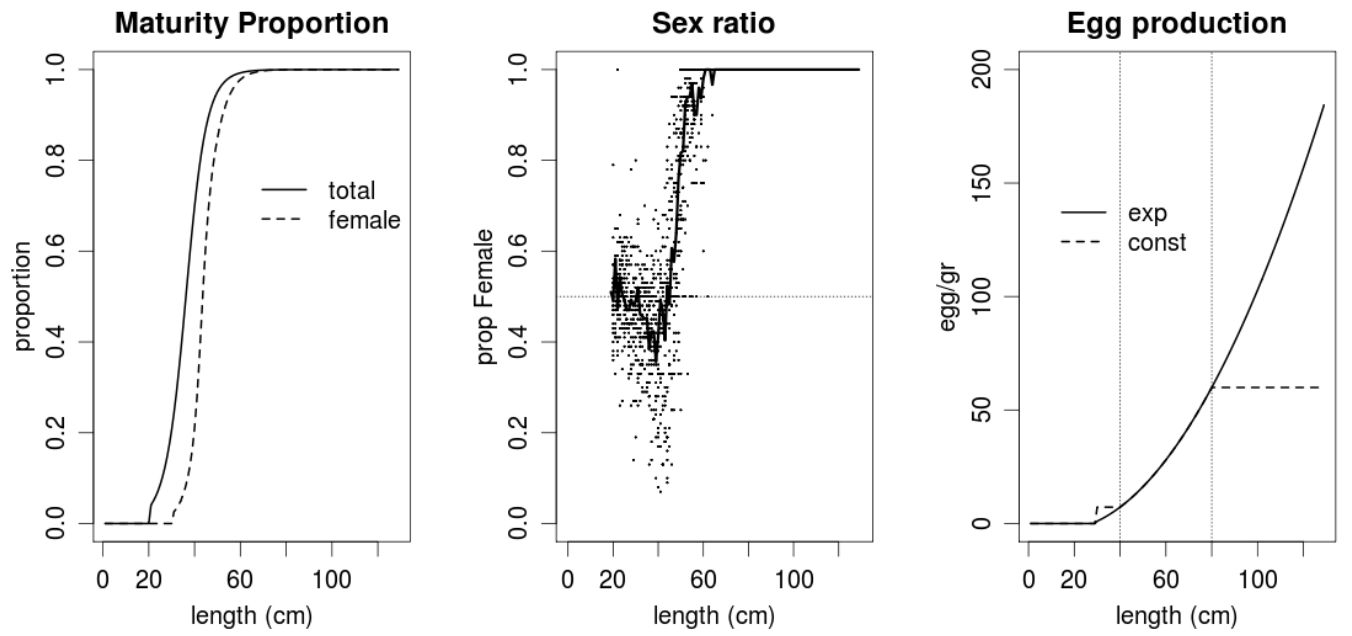
Figure 2
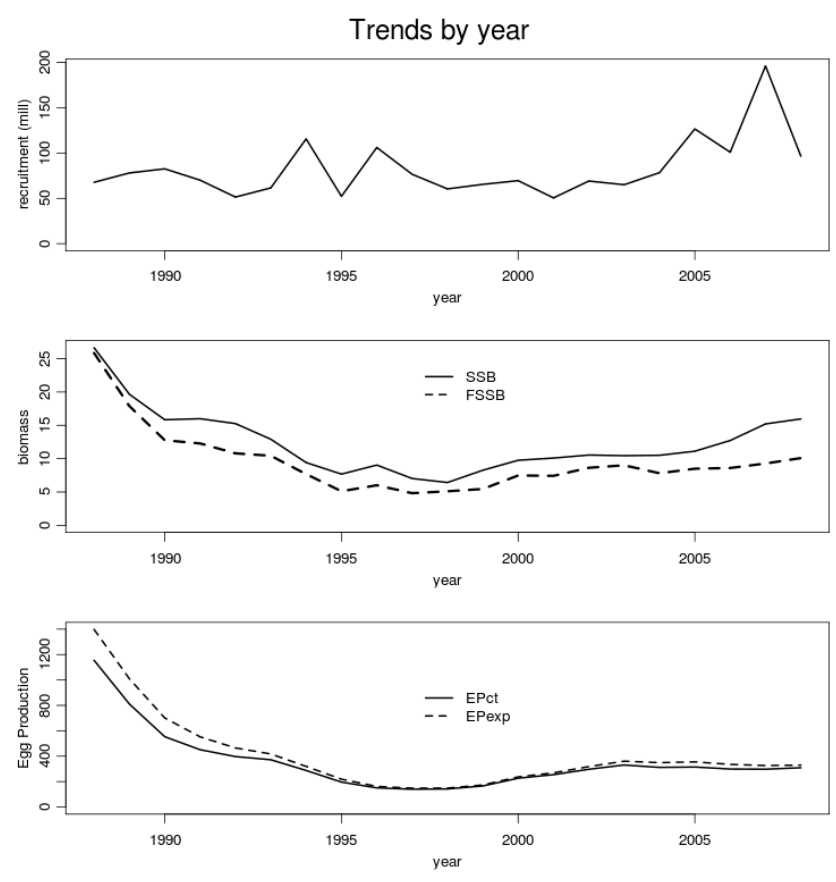
Figure 3

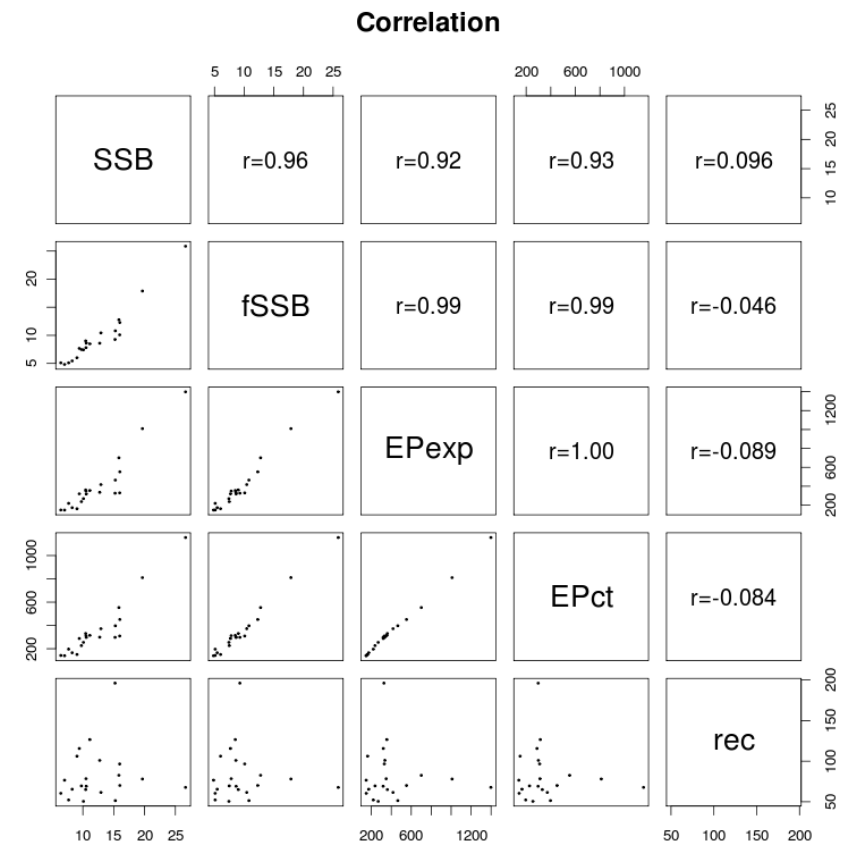


Figure 4

Recruitment predicted $[0.05,0.5,0.95]$
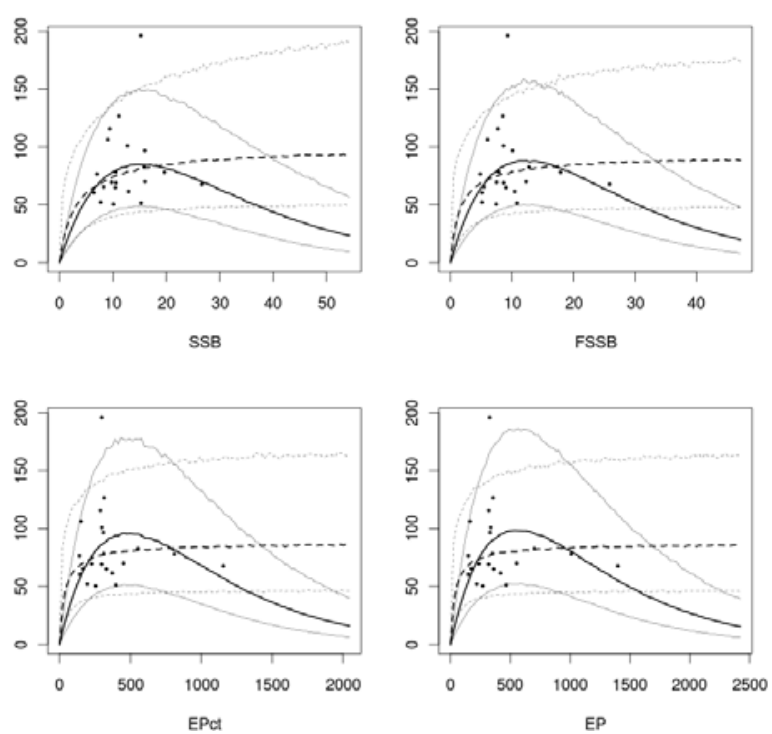
Figure 5

Ricker Steepness
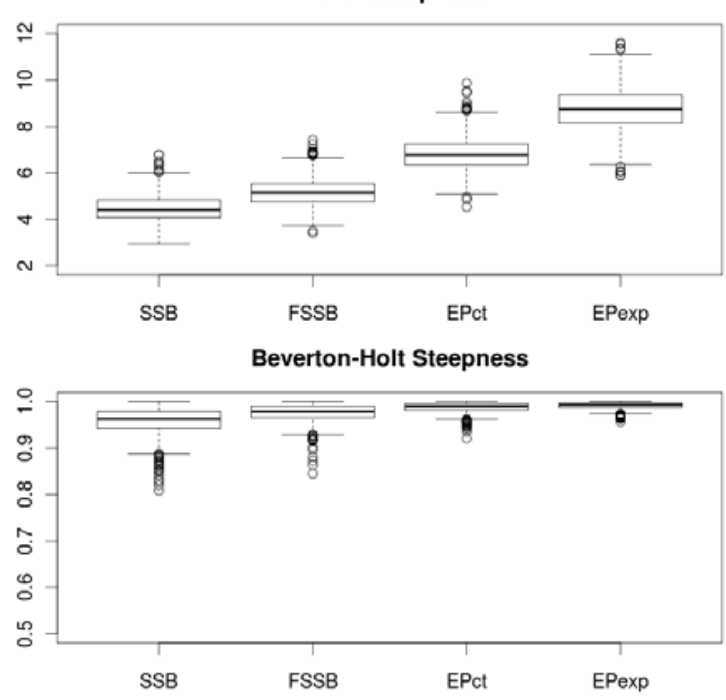
Figure 6

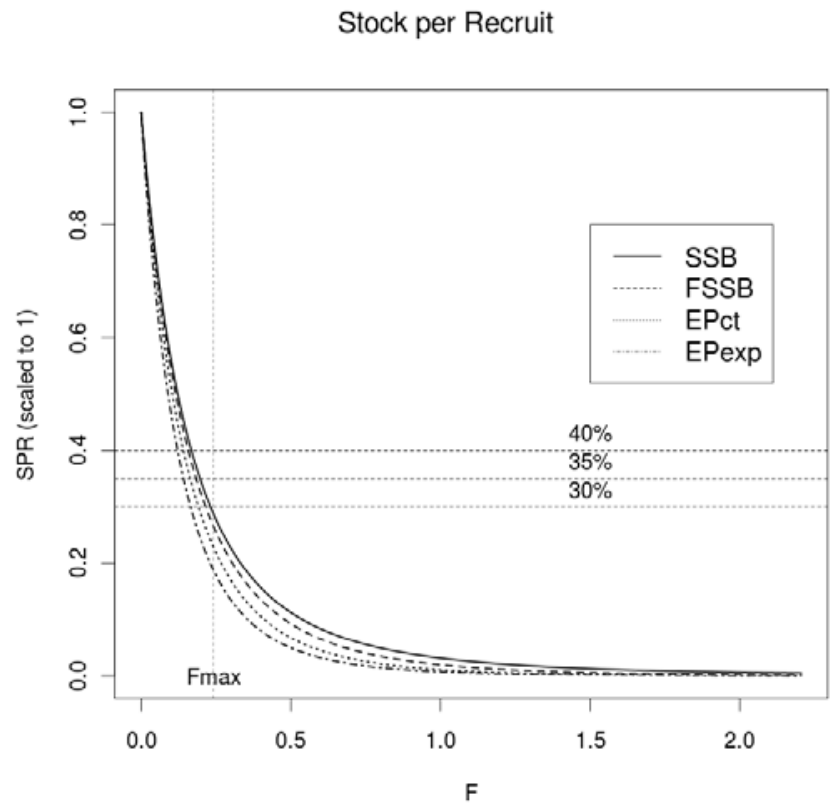


Figure 7

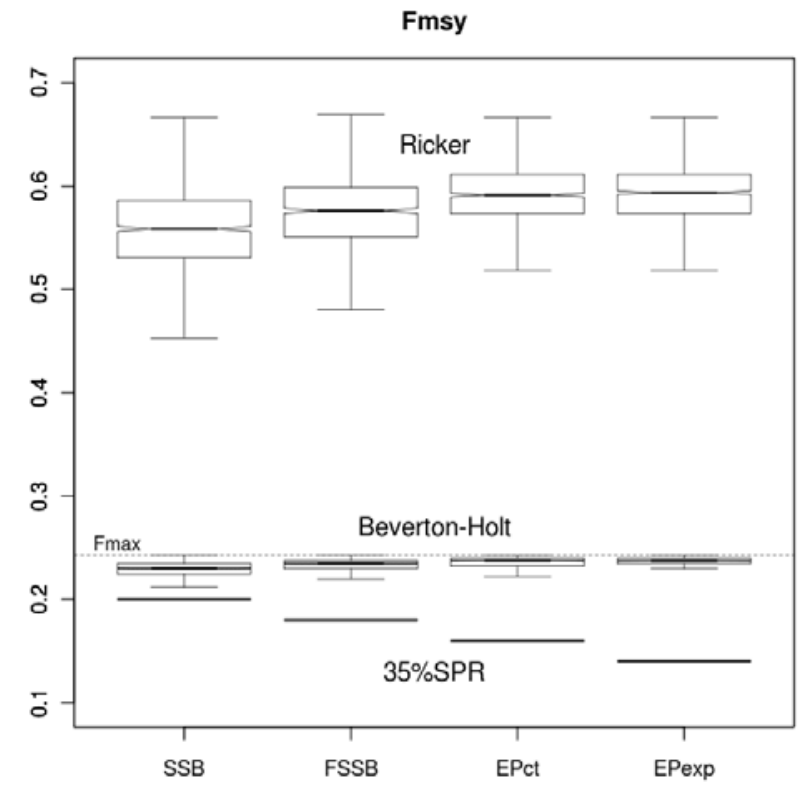


Figure 8
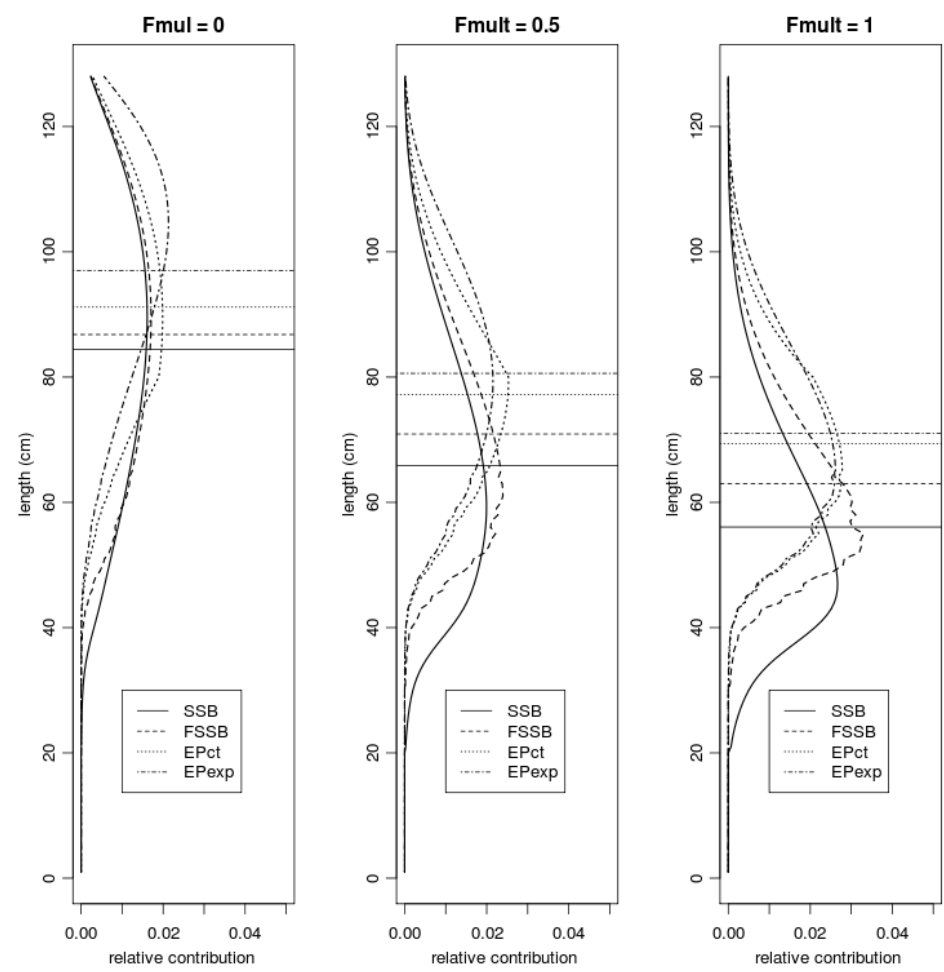